\title{
Adaptive fading factor unscented Kalman filter with application to target tracking
}

\author{
Peng Gu ${ }^{1,2} \cdot$ Zhongliang Jing ${ }^{1} \cdot$ Liangbin $\mathrm{Wu}^{2}$
}

Received: 6 July 2020 / Revised: 16 October 2020 / Accepted: 23 October 2020 / Published online: 17 November 2020

(c) The Author(s) 2020

\begin{abstract}
One purpose of target tracking is to estimate the states of targets, and unscented Kalman filter is one of the effective algorithms for estimating in the nonlinear tracking problem. Considering the characteristics of complex maneuverability, it is easy to reduce the tracking accuracy and cause divergence due to the mismatch between the system model and the practical target motion model. Adaptive fading factor is an effective counter to this problem, having been instrumental in solving accuracy and divergence problems. Fading factor can adaptively adjust covariance matrix online to compensate model mismatch error. Moreover, fading factor not only improves the filtering accuracy, but also automatically adjusts the error covariance in response to the different situation. The simulation results show that the adaptive fading factor unscented Kalman filter has more advantages in target tracking and it can be better applied to nonlinear target tracking.
\end{abstract}

Keywords Adaptive fading factor · Target tracking · UKF · Accuracy

\section{Introduction}

Kalman filter algorithm can derive the optimal estimation of state under conditions involving linear-Gaussian assumption, which is based on the known system model, observation model and statistics of noises. When they are inconsistent with target behavior model, the estimation error will increase. Singer model can express the acceleration of maneuver as a time-dependent process [1], which conforms to actual target motion. By adjusting the frequency coefficient of maneuvering to achieve better tracking effect, it has been widely used for nonlinear system $[2,3]$. The "current" statistical model was proposed as a representative of adaptive tracking algorithm [4]. In this model, the acceleration noise is assumed to

\section{Peng Gu}

gpszseu@163.com

Zhongliang Jing

zljing@sjtu.edu.cn

Liangbin $\mathrm{Wu}$

lbwu0105@126.com

1 School of Aeronautics and Astronautics, Shanghai Jiao Tong University, Shanghai, China

2 AVIC Leihua Electronic Technology Research Institute, Wuxi, China be Rayleigh distribution, and the distribution of state noise is updated in real time through mean value of acceleration.

In the process of target tracking, if the system model deviates from their actual values by unknown random bias, the virtual noise is usually used to reduce confidence level of the filter to the system model, but it is difficult to determine how much virtual noise to be added. At this time, we should consider abandoning the previous observation data, and pay more attention to the newly generated observation value, so that the filter has better performance. Researchers have proposed a variety of solutions for this problem. The adaptive fading Kalman filter (AFKF) $[13,14]$ uses the fading factor to suppress the memory length of the filter, so as to make full use of the new measurement and reduce the influence of the old measurement. However, the expressions of these methods are complex; so, they have real-time performance problems. Thus, this paper proposes a new method for overcoming the problem to obtain the fading factor. In this method, the covariance of the innovation is used to adaptively change the fading factor which adjusts the weight of innovation in real time, so as to reduce the influence of the old measurement on the estimation. In this paper, the fading factor is integrated into unscented Kalman filter to form adaptive fading factor unscented Kalman filter (AFUKF). In general, we can put weight of fading factor on the prediction covariance matrix, so that the output value of the estimation is 
closer to the actual value. Meanwhile, the adaptive method of the fading factor is described in detail throughout this paper.

\section{The unscented Kalman filter}

For the general nonlinear maneuvering target tracking, techniques for nonlinear filtering typically involve classic estimation methods such as extended Kalman filter (EKF) [5] and Quadrature Kalman filter (QKF) [6] which is approximated by Gauss Hermite quadrature criterion. When the nonlinearity of the system is too severe, piecewise linear application of EKF degrades performance to the nonlinear tracking problem. The extended Kalman filter (EKF) propagates mean value through the linearization of the nonlinear conditions. As the EKF uses the first-order terms as an approximation, linearization errors will rise if neglected higher-order terms begin to dominate. However, sampling points of QKF will grow exponentially as the spatial dimension increases. Thus, QKF is certainly not suitable for high-dimensional cases.

The core of unscented Kalman filter (UKF) is UT transformation, which is a method for calculating the statistics value of random variable to avoid nonlinear transformation $[7,8]$. The fundamental idea is found on the intuition that it is easier to approximate the probability distribution and statistical variables with finite variables than it is to approximate a nonlinear transformation $[9,10]$. The principle of UT transformation is that a set of points (sigma points) are selected according to a certain rule in the original state distribution. This makes the mean and covariance of these points equal to the mean and covariance of the original state distribution. When the sigma points are put into state equation and observation equation, the statistics can be obtained from small scale nonlinear transformations. The optimal state estimates and their covariance matrix can be derived using the statistics of the transformed points [11]. The estimation process is summarized in Table 1 for one cycle.

Repeating the cycle for the next time, the current state estimation and covariance matrix are obtained as input sources to the estimation processes.

Here, $\hat{x}_{i}(k \mid k-1)$ is the one-step predicted state, $f(\cdot)$ is the nonlinear state function, $Q_{k}$ is the Gaussian process noise, $R_{k}$ is the Gaussian measurement noise, $h(\cdot)$ is the measurement function, $\lambda$ is the adjustable scalar. $P(k \mid k-$ $1)$ is the one-step predicted covariance, $\sqrt{(n+\lambda) P(k \mid k-1)}$ is the $i$ th row or column of the matrix square root of $(n+\lambda) P(k \mid k-1), P_{z z}(k \mid k-1)$ is the predicted innovation covariance, $P_{x z}(k \mid k-1)$ is the predicted cross-correlation matrix, $W_{i}$ is the weight that is associated with the $i$ th point, $\Gamma_{i}$ is a scalar.

\section{Adaptive fading filter method}

\subsection{Fading memory method}

When the system model is not accurate or mismatch arises between system model and reality, the current observations only play a small role in correcting the estimation. While the previous observations cause the tracking error and filter divergence, the fading memory algorithm is to redistribute the current observation and improve the robustness of the filter:

$$
\begin{aligned}
& P_{x z}(k \mid k-1)=\alpha \sum_{i=0}^{2 N} W_{i}\left(\hat{x}_{i}(k \mid k-1)\right. \\
& \quad-\hat{x}(k \mid k-1))\left(\zeta_{i}(k \mid k-1)-\hat{z}(k \mid k-1)\right)^{T}+Q_{k}=\hat{P}+Q_{k} .
\end{aligned}
$$

Assume that $\alpha=1$, the fading memory algorithm is equivalent to the unscented Kalman filter, where $\hat{P}$ is the state error covariance in the ideal situation where the system model completely matches the moving target, i.e., with $Q_{k}=0$. At this time, the fading factor can alter the coefficient of the state covariance according to the current observation data. The deviation of higher degree between the system model and actual target state can make the coefficient larger. To compensate for the estimation error caused by the model mismatch, the observations must be given a higher degree of confidence level. When $\alpha$ is too large, the estimation eventually converges to the observation value which may cause the loss of accuracy of the state estimation.

\subsection{Adaptive fading factor method}

The actual error between target state and prediction model results in model mismatch. The near measurement contains more dynamic model information of targets; thus, the new measurement information mainly corrects state estimation error effects to improve the filtering robustness. In this situation, the fading factor are introduced into unscented Kalman filter, where $\rho$ is a prior coefficient, $\alpha_{k}$ calculation involves the following formulae [12]:

$$
\begin{aligned}
& S_{k+1}=\left\{\begin{array}{l}
v_{1} v_{1}^{T} k=0 \\
\frac{\rho S_{k}+v_{k+1} v_{k+1}^{T}}{1+\rho} k \geq 1,
\end{array}\right. \\
& N=S_{k+1}-R, M=P_{z z}-R, \\
& \lambda_{k}=\frac{\operatorname{trace}(N)}{\operatorname{trace}\left(P_{z z}(k \mid k-1)\right)}, \\
& \alpha_{k}= \begin{cases}\lambda_{k} & \lambda_{k} \geq 1 \\
1 & \lambda_{k}<1 .\end{cases}
\end{aligned}
$$


Table 1 The unscented Kalman filter

$$
\begin{aligned}
& \text { Prediction stage } \\
& \text { For } i=1: L \\
& \hat{x}_{i}(k \mid k-1)=f\left(\hat{x}(k-1)+\Gamma_{i} \sqrt{(n+\lambda) P_{i}(k-1)}\right) \\
& \text { End } \\
& \hat{x}(k \mid k-1)=\sum_{i=0}^{2 N} W_{i} \hat{x}_{i}(k \mid k-1) \\
& P(k \mid k-1)=\sum_{i=0}^{2 N} W_{i}\left(\hat{x}_{i}(k \mid k-1)-\hat{x}(k \mid k-1)\right)\left(\hat{x}_{i}(k \mid k-1)-\hat{x}(k \mid k-1)\right)^{T}+Q_{k} \\
& \text { Update stage } \\
& \text { For } i=1: L \\
& \hat{x}_{i}(k \mid k-1)=\hat{x}(k \mid k-1)+\Gamma_{i} \sqrt{(n+\lambda) P(k \mid k-1)} \\
& \zeta_{i}(k \mid k-1)=h\left(\hat{x}_{i}(k \mid k-1)\right) \\
& \text { End } \\
& \hat{z}(k \mid k-1)=\sum_{i=0}^{2 N} W_{i} \zeta_{i}(k \mid k-1) \\
& P_{z z}(k \mid k-1)=\sum_{i=0}^{2 N} W_{i}\left(\zeta_{i}(k \mid k-1)-\hat{z}(k \mid k-1)\right)\left(\zeta_{i}(k \mid k-1)-\hat{z}(k \mid k-1)\right)^{T}+R_{k} \\
& P_{x z}(k \mid k-1)=\sum_{i=0}^{2 N} W_{i}\left(\hat{x}_{i}(k \mid k-1)-\hat{x}(k \mid k-1)\right)\left(\zeta_{i}(k \mid k-1)-\hat{z}(k \mid k-1)\right)^{T} \\
& K(k)=P_{x z}(k \mid k-1) P_{z z}^{-1}(k \mid k-1) \\
& \hat{x}(k \mid k)=\hat{x}(k \mid k-1)+K(k)(z(k)-\hat{z}(k \mid k-1)) \\
& P(k \mid k)=P(k \mid k-1)-K(k) P_{z z}(k \mid k-1) K(k)^{T}
\end{aligned}
$$

The innovation covariance reflects the effect of the current error. The innovation covariance and predicted state covariance will increase due to the mismatch model. According to Eq. (1), the innovation covariance adjusted by $\alpha_{k}$ can be regarded as the prediction state covariance adjusted by $\lambda_{k}$. Therefore, the effect of mismatch information in dynamic system can be compensated by adjusting the value of $P_{x z}(k \mid k-1)$. Covariance $P_{z z}$ can be used as an evaluation criterion to judge the uncertainty of state prediction in the process of filtering. Meanwhile, $v_{k+1}$ is prediction residual error that can be obtained from observation, corresponding to the error of state estimation. It can be seen that whether covariance divergence is likely to occur can be determined by the value of $\alpha_{k}$. It demonstrates that the real-time covariance of state estimation is larger than the covariance of state prediction when $\alpha_{k}>1$. At this time, we need to make some adjustments to the system model. The weight of the current observation can be increased by the fading memory algorithm. According to the property description of $S_{k+1}$, adaptive method of fading factor for UKF called AFUKF algorithm can be established to diminish error covariance when the estimation error is caused by model mismatch. The adaptive fading factor unscented Kalman filter is summarized in Table 2.

Repeating the cycle for the next time, the current state estimation and covariance matrix are obtained as input sources to the estimation processes.
Here, $\hat{x}_{i}(k \mid k-1)$ is the one-step predicted state, $f(\cdot)$ is the nonlinear state function, $Q_{k}$ is the Gaussian process noise, $R_{k}$ is the Gaussian measurement noise, $h(\cdot)$ is the measurement function, $\lambda$ is the adjustable scalar. $P(k \mid k-$ $1)$ is the one-step predicted covariance, $\sqrt{(n+\lambda) P(k \mid k-1)}$ is the $i$ th row or column of the matrix square root of $(n+\lambda) P(k \mid k-1), P_{z z}(k \mid k-1)$ is the predicted innovation covariance, $P_{x z}(k \mid k-1)$ is the predicted cross-correlation matrix, $W_{i}$ is the weight that is associated with the $i$ th point and $\Gamma_{i}$ is a scalar.

\section{Simulation}

The proposed AFUKF is applied to the target tracking system as compared to given UKF algorithm and Sage-UKF algorithm. The radar observation station is set at the origin of coordinate in space of two-dimensional scenario, where the target makes the approximate S-type maneuver. The nonlinear system equation and observation equation of the target motion are described as follows:

$$
\begin{aligned}
& x_{k+1}=\left(\begin{array}{ccc}
1 & T & \left(\alpha T-1+e^{-\alpha T}\right) / \alpha^{2} \\
0 & 1 & \left(1-e^{-\alpha T}\right) / \alpha \\
0 & 0 & e^{-\alpha T}
\end{array}\right) x_{k}+Q_{k}, \\
& z_{k}=\left[\begin{array}{l}
\sqrt{x_{k}^{2}+y_{k}^{2}} \\
\arctan \left(y_{k} / x_{k}\right)
\end{array}\right]+\left[\begin{array}{c}
v_{k}^{r} \\
v_{k}^{a}
\end{array}\right],
\end{aligned}
$$


Table 2 The adaptive fading factor unscented Kalman filter

$$
\begin{aligned}
& \text { Prediction stage } \\
& \text { For } i=1: L \\
& \hat{x}_{i}(k \mid k-1)=f\left(\hat{x}(k-1)+\Gamma_{i} \sqrt{(n+\lambda) P_{i}(k-1)}\right) \\
& \text { End } \\
& \hat{x}(k \mid k-1)=\sum_{i=0}^{2 N} W_{i} \hat{x}_{i}(k \mid k-1) \\
& P(k \mid k-1)=\sum_{i=0}^{2 N} W_{i}\left(\hat{x}_{i}(k \mid k-1)-\hat{x}(k \mid k-1)\right)\left(\hat{x}_{i}(k \mid k-1)-\hat{x}(k \mid k-1)\right)^{T}+Q_{k} \\
& \text { Update stage } \\
& \text { For } i=1: L \\
& \hat{x}_{i}(k \mid k-1)=\hat{x}(k \mid k-1)+\Gamma_{i} \sqrt{(n+\lambda) P(k \mid k-1)} \\
& \zeta_{i}(k \mid k-1)=h\left(\hat{x}_{i}(k \mid k-1)\right) \\
& \text { End } \\
& \hat{z}(k \mid k-1)=\sum_{i=0}^{2 N} W_{i} \zeta_{i}(k \mid k-1) \\
& P_{z z}(k \mid k-1)=\sum_{i=0}^{2 N} W_{i}\left(\zeta_{i}(k \mid k-1)-\hat{z}(k \mid k-1)\right)\left(\zeta_{i}(k \mid k-1)-\hat{z}(k \mid k-1)\right)^{T}+R_{k} \\
& P_{x z}(k \mid k-1)=\alpha_{k} \sum_{i=0}^{2 N} W_{i}\left(\hat{x}_{i}(k \mid k-1)-\hat{x}(k \mid k-1)\right)\left(\zeta_{i}(k \mid k-1)-\hat{z}(k \mid k-1)\right)^{T} \\
& K(k)=P_{x z}(k \mid k-1) P_{z z}^{-1}(k \mid k-1) \\
& \hat{x}(k \mid k)=\hat{x}(k \mid k-1)+K(k)(z(k)-\hat{z}(k \mid k-1)) \\
& P(k \mid k)=P(k \mid k-1)-K(k) P_{z z}(k \mid k-1) K(k)^{T} \\
& S_{k}=\frac{\rho S_{k-1}+v_{k} v_{k}^{T}}{1+\rho} \\
& N=S_{k}-R, M=P_{z z}-R \\
& \lambda_{k}=\frac{\operatorname{trace}(N)}{\operatorname{trace}\left(P_{z z}(k \mid k-1)\right)} \\
& \alpha_{k}=\left\{\begin{array}{l}
\lambda_{k} \lambda_{k} \geq 1 \\
1 \lambda_{k}<1
\end{array}\right.
\end{aligned}
$$

\begin{tabular}{lllll}
\hline Method & Position $(\mathrm{m})$ in $X$ & Position $(\mathrm{m})$ in $Y$ & Velocity $(\mathrm{m} / \mathrm{s})$ in $X$ & Velocity $(\mathrm{m} / \mathrm{s})$ in $Y$ \\
\hline UKF & 289.99 & 402.62 & 60.02 & 101.46 \\
SAGE-UKF & 275.05 & 383.97 & 49.60 & 91.22 \\
AFUKF & 205.45 & 314.99 & 18.93 & 55.89 \\
\hline
\end{tabular}

Table 2 Comparison of RMS for different methods

$$
q_{13}=q_{31}=\frac{1}{2 \alpha^{3}}\left(1-e^{-2 \alpha T}-2 \alpha T e^{-\alpha T}\right),
$$
and azimuth noise of the observation, respectively, $Q_{k}$ is the process noise:

$Q_{k}=2 \alpha \sigma^{2}\left(\begin{array}{lll}q_{11} & q_{12} & q_{13} \\ q_{21} & q_{22} & q_{23} \\ q_{31} & q_{32} & q_{33}\end{array}\right)$

(8) $\quad q_{22}=\frac{1}{2 \alpha^{3}}\left(-3+4 e^{-\alpha T}-e^{-2 \alpha T}+2 \alpha T\right)$,

where

$$
\begin{aligned}
& q_{11}=\frac{1}{2 \alpha^{5}}\left(1-e^{-2 \alpha T}+2 \alpha T+\frac{2 \alpha^{3} T^{3}}{3}-2 \alpha^{2} T^{2}-4 \alpha T e^{-\alpha T}\right), \\
& q_{12}=q_{21}=\frac{1}{2 \alpha^{4}}\left(1+e^{-2 \alpha T}-2 e^{-\alpha T}+2 \alpha T e^{-\alpha T}-2 \alpha T+\alpha^{2} T^{2}\right), \quad q_{33}=\frac{1}{2 \alpha}\left(1-e^{-2 \alpha T}\right),
\end{aligned}
$$




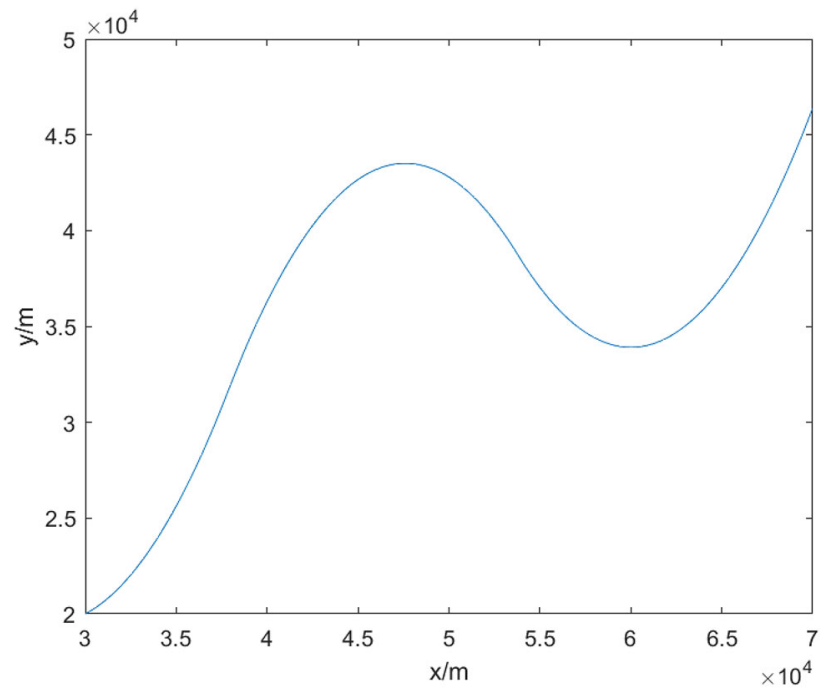

Fig. 1 Real target trajectory

where $\alpha=1$ is the frequency of maneuvering, $\sigma=1$ is the scale factor, $T=1$ is the sampling period. $R_{k}$ is the observation noise.

$R_{k}=\left(\begin{array}{cc}r^{2} & 0 \\ 0 & a z^{2}\end{array}\right)$

where $r=60, a z=0.02$.

As shown in Fig. 1, the flight path is used for simulation analysis. In the rectangular coordinate system, the target position can be regarded as the input state of the system. The vector $s_{0}=\left[x_{0}, y_{0}\right]$ is used to describe the initial target position, where $x_{k}$ and $y_{k}$ represent the horizontal position and vertical position of the target, respectively, the initial position of the target is $s_{0}=[30,000,20,000][30,000,20,000]$, the initial velocity and acceleration can be derived from the position and time using two-point differencing method.

Root mean square (RMS) tracking error provides a quantitative estimate of how close the target position actually is to the true target position. The performance of each of the algorithms was assessed using 50 Monte Carlo runs, which are shown in Figs, 2, 3, 4 and 5. The system model deviates so far from the actual motion model when the target turns suddenly; the AFUKF performs better than the traditional methods which rapidly degrades the accuracy of the estimate. This experiment verifies the feasibility of adaptive fading factor unscented Kalman filter in practical application, and the $\alpha_{k}$ value changes with the mismatch model error, which can achieve the real-time identification effect.

The AFUKF algorithm proposed in this paper uses the fading factor to adjust the statistical properties of the system noise in real time for estimating target states. It can effectively solve the problem that the estimation error increases due to the mismatch between the system model and the actual

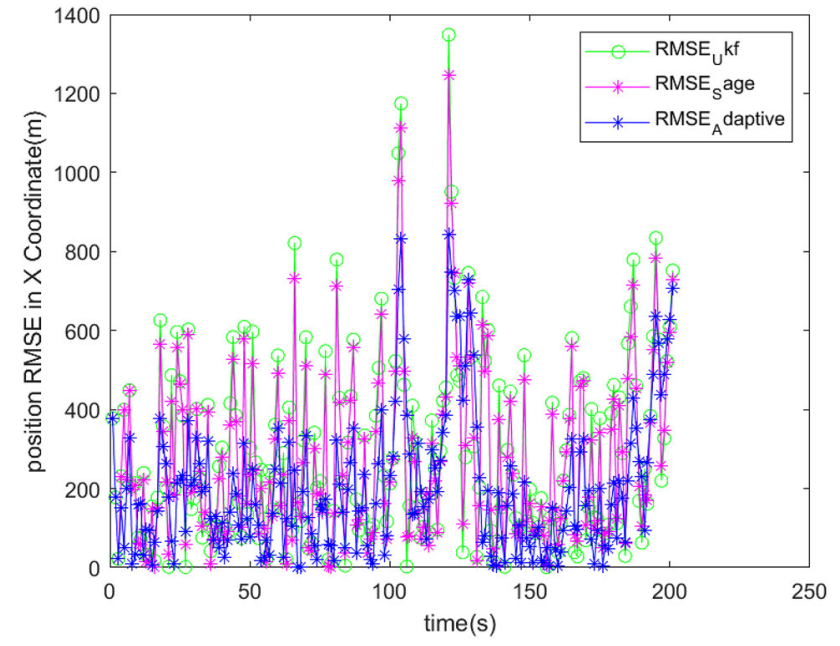

Fig. 2 The position RMSE in $X$ coordinate for different methods over time

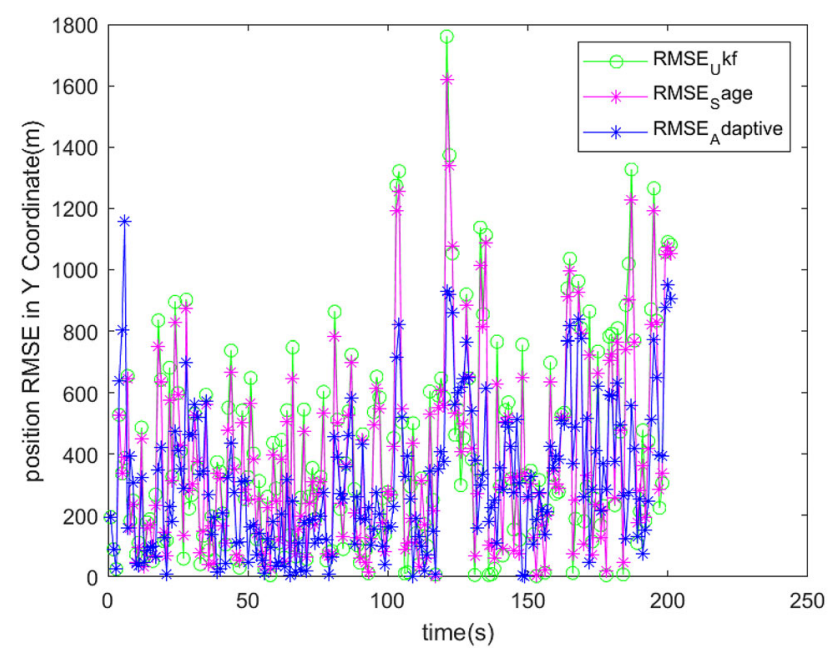

Fig. 3 The position RMSE in $Y$ coordinate for different methods over time

model to ensure a quicker convergence and enhance the accuracy of estimation. As shown in Table 2, the RMS errors of position and velocity of the AFUKF algorithm are significantly smaller than those of standard algorithms. Simulation results show that the AFUKF algorithm is significantly better than the standard UKF Algorithm in accuracy and stability of estimation, and the state estimation is also closer to the real target state.

\section{Conclusion}

This paper describes the problem of mismatch between the system model and the practical target motion model when target enters the maneuver in tracking. To solve this problem, adaptive fading factor unscented Kalman filter algorithm is 


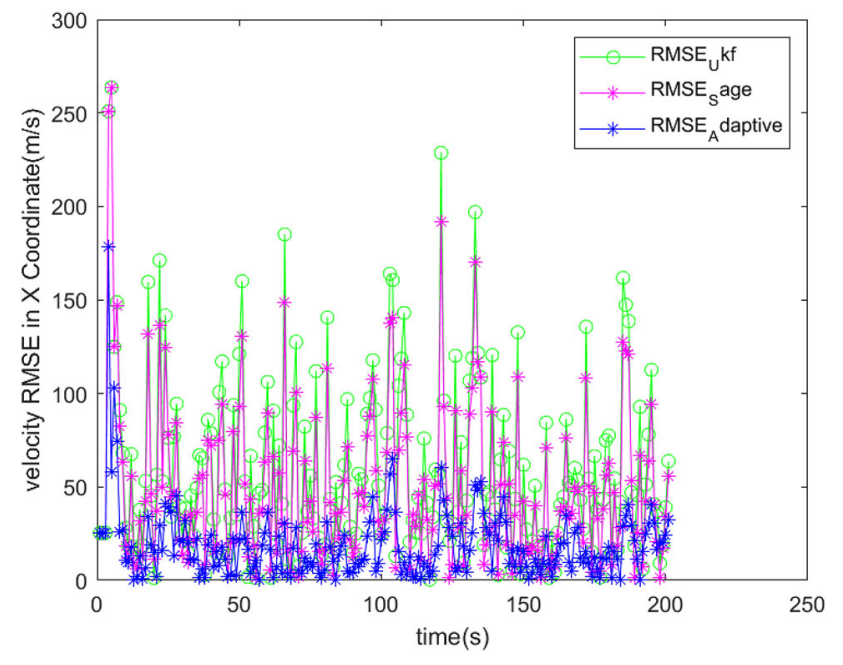

Fig. 4 The velocity RMSE in $X$ coordinate for different methods over time

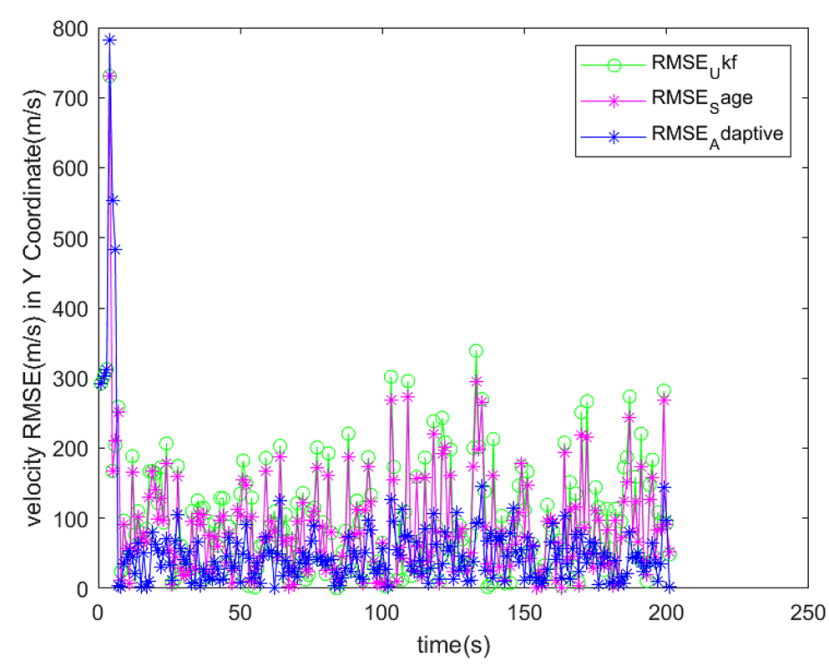

Fig. 5 The velocity RMSE in $Y$ coordinate for different methods over time

proposed to reduce the errors of estimation. The method of obtaining fading factor by innovation covariance is simple in calculation, and it avoids a large number of numerically intensive calculations. It overcomes some problems in traditional calculation method and can be used in complex multi-target tracking scenario. In addition, the algorithm demonstrates the feasibility of adaptive fading factor from the perspective of prediction covariance and actual covariance to avoid the calculation of Jacobian matrix of nonlinear and the deviation caused by the first-order linearization. Simulation results show that the proposed method is an effective way to improve the tracking accuracy than the traditional methods.

Acknowledgements This work was supported in part by the National Natural Science Foundation of China under Grant 61673262 and Grant
61175028, and in part by the Shanghai key Project of Basic Research under Grant 16JC1401100.

Open Access This article is licensed under a Creative Commons Attribution 4.0 International License, which permits use, sharing, adaptation, distribution and reproduction in any medium or format, as long as you give appropriate credit to the original author(s) and the source, provide a link to the Creative Commons licence, and indicate if changes were made. The images or other third party material in this article are included in the article's Creative Commons licence, unless indicated otherwise in a credit line to the material. If material is not included in the article's Creative Commons licence and your intended use is not permitted by statutory regulation or exceeds the permitted use, you will need to obtain permission directly from the copyright holder. To view a copy of this licence, visit http://creativecomm ons.org/licenses/by/4.0/.

\section{Referencess}

1. Singer RA (1970) Estimating optimal filter tracking performance for manned maneuvering targets. IEEE Trans Aerosp Electron Syst $6(4): 473-483$

2. Uhlmann JK (1992) Algorithms for multiple target tracking. Am Sci 80(2):128-141

3. Crassidis JL, Markley FL, Cheng Y (2007) Survey of nonlinear attitude estimation methods. J Guid Control Dyn 30(1):12-28

4. Kumar KSP, Zhou H (1984) A "current" statistical model and adaptive algorithm for estimating maneuvering targets. AIAA J Guid 7(5):596-602

5. Julier SJ, Uhlmann JK (1999) A new extension of the Kalman filter to nonlinear systems. Proc SPIE Int Soc Opt Eng 3068:182-193

6. Closas P, Fernandez-Prades P, Vila-Valls P (2012) Multiple quadrature Kalman filtering. IEEE Trans Signal Process 60(12):6125-6137

7. Julier SJ, Uhlmann JK, Durrant-Whyte HF (1995) A new approach for filtering nonlinear systems. In: American control conference, pp $1628-1632$

8. Julier SJ, Uhlmann JK (2003) The spherical simplex unscented transformation. In: American control conference, pp 2430-2434

9. Crassidis JL, Markley FL (2003) Unscented filtering for spacecraft attitude estimation. J Guid Control Dyn 26(3):536-542

10. Rao S, Rajeswari K, Lingamurty K (2009) Unscented Kalman filter with application to bearings-only target tracking. Iete J Res 55(2):63

11. Sorenson HW, Sacks JE (1971) Recursive fading memory filtering. Inf Sci 3(2):101-119

12. Soken HE, Hajiyev C (2011) Adaptive fading UKF with Qadaptation: application to picosatellite attitude estimation. J Aerosp Eng 26(3):628-636

13. Lee TS (1988) Theory and application of adaptive fading memory Kalman filters. IEEE Trans Circuits Syst 35(4):474-477

14. Xia QJ, Rao M, Ying YQ (1994) Adaptive fading Kalman filter with an application. Automatica 30(8):1333-1338 